\title{
Research and Application of Failure Detection Method Based on Closed-loop Systems
}

\author{
Li-Fei Deng ${ }^{1}$, Yaowu Shi ${ }^{1}$, Lan-Xiang Zhu ${ }^{2 *}$, D. L. Yu ${ }^{3}$, Rui Zhu ${ }^{4}$ \\ ${ }^{1}$ College of Communication Engineering, Jilin Univ. , Changchun 132013, China \\ ${ }^{2}$ Changchun Architecture And Civil engineering College, Changchun 130069, \\ China \\ ${ }^{3}$ Control Group, School of Engineering, Liverpool John Moores University, \\ Liverpool, U.K. \\ ${ }^{4}$ Changchun zonbo new energy transmission system of limited liability company, \\ China \\ Corresponding author: 519007262@qq.com
}

\begin{abstract}
When a neural network model is trained to predict system output, the prediction error can be used as residual to report fault. Most existing research uses system open-loop input/output data, while the trained model is used to detect fault when the system runs under closed-loop control. This paper analyses the drawback of the training data acquisition and proposes new data acquisition method, so that the model accuracy is greatly improved. In addition, detection of the sensor fault, which is involved in the closed-loop, is discussed and the simulation for detecting such sensor faults are conducted. The new scheme is assessed and validated by being applied to the automotive engine air path to detect some simulated faults. The simulation results show that the developed method is effective and the residual is more sensitive to the faults
\end{abstract}

Keywords: Fault detection, automotive engines, closed-loop fault detection, independent RBF model.

\section{Introduction}

Although Fault detection and isolation (FDI) for automotive engines have been investigated for over two decades, but real applications with on-board FDI for dynamic faults (not the faults occurred during the steady state) have seldamly reported. There are two main reasons. One is that the false alarm rate is too high to be acceptable; the other is that most methods are too complecated and need accurate mathematical model, which is not practical to implement.

The typical work that has done before is briefly introduced here. Gertler [1-2] and his co-workers attemted using linear Parity space method to detect simulated faults. Simulated faults and experiments have been done. But the results were not very satisfactory due to the severe nonlinearities in engine dynamics. Reference [3] introduced an electro-mechanical position servo, used in the speed control of large diesel engines, as a benchmark for model based fault detection and identification. Isermann [4] proposed model-based fault-detection and diagnosis methods for some technical processes. The goal was to generate several symptoms indicating the difference between nominal and faulty status. On-line sensor fault detection, isolation and accommodation in automotive engines have been studied by Capriglione, [5-7]. Their paper described the hybrid solution, based on artificial neural networks (ANN), but their methods used ANN just as classifiers rather than 
dynamic models. Therefore, the application of the method would not be straight forward and need adjustment for each individual engine.

It is noticed in the literature that most reported research on FDI of open-loop internal combustion engines. And the FDI methods developed on the open-loop engines will find difficulties and problems when they are tested with the engines under closed-loop control. In further, these problems also appear when these methods were tried to be applied to real engine systems. The main differences between open-loop engine and engine under closed-loop control are lies on the following points.

Firstly, the neural network model of the engine air path needs to be trained with engine input/output data. In order to excite the engine dynamics on the whole range of frequency, for open-loop engine a random amplitude sequence (RAS) is used. However, as the input signal for engine under closed-loop control is the controller output and cannot be designed, the persistent exciting will not be achieved. One method is to superimpose a pseudorandom binary sequence (PRBS) onto the control signal. To excite nonlinear dynamics of a nonlinear dynamic system such as engines, both amplitude and frequency of the excitation signal should be considered for their wide range. As the diverged amplitude can be achieved by the variable amplitude of the control signal, while the diverged frequency achieved by the PRBS, the above stated signal will be used in this research.

Secondly, the RAS signal applied to set-point of the closed-loop system has been considered in this research, and then the generated control signal is applied to the engine for data excitation. This is from the fact that in practice the disturbance, the throttle angle change, is often a step change, which can be simulated by a RAS signal. On other hand, the control signal would be a filtered signal (as the controller could be seen as a filter) and lost the deep edge of the step signal, so the high frequency part would be lost. However, in practice it is just this type of signal dominated the operation process, and therefore, the training of the NN model with these data would be reasonable.

Thirdly, the output of some sensors may be used as feedback signal in the closedloop control. This will change the signal pattern completely. When a sensor fault occurs, the sensor output is feedback to change the error signal, so that the engine output is regulated to a value such that the sensor output nearly equivalent to the set-point. This makes the deviation of the sensor output from nominal value quickly vanishes, and consequently the detection on this signal would become more difficult.

In this paper, a new fault detection and isolation method will be implemented by using mean value engine model when this model is under close loop control system. An independent RBFNN model is used to model a dynamic system. Feed-back (FB) and feed-forward (FF) control methods will be applied to the mean value engine model. Firstly, fuel injection value corresponding to angle position value will be defined by using MVEM. Secondly, PID controller will be used in the feed-back to adjust the difference between the requested and the actual air fuel ratio by compensating the feed-forward controller output[9]. Thirdly RBF neural network models will be used to predict MVEM outputs. The model prediction error is used to generate residual for fault detection. Fault isolation will not be discussed in this paper due to the limited space. The proposed method is applied to air path dynamics of the IC engines using a well-know benchmark simulation model: mean value engine model (MVEM) [8]. Different types of fault were simulated on the MVEM, and then the input/output data were used to detect and isolate these faults. 


\section{Engine Dynamics}

In this work, the fault detection method is developed and evaluated based on the Mean Value Engine Model (MVEM) [8] of which a block diagram is shown in Figure 1.

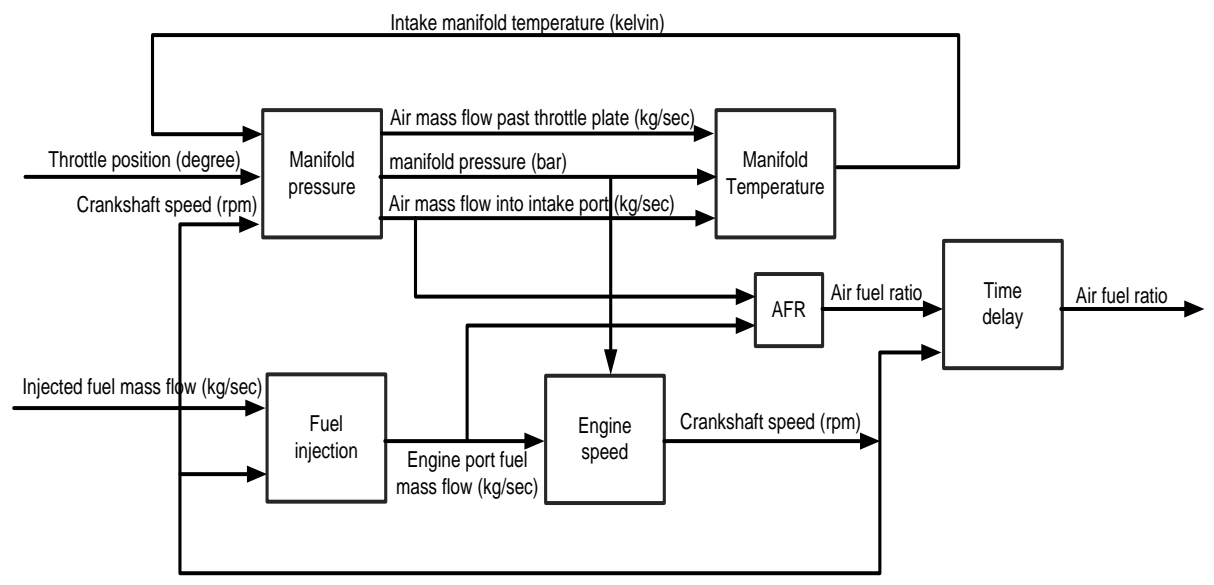

Figure 1. Mean Value Engine Model

A. Manifold Filling Dynamics

It includes two nonlinear differential equations: one for the manifold pressure and the other for the manifold temperature. The manifold pressure is described as

$$
\dot{p}_{i}=\frac{\mathbb{P N}_{\mathbb{P}}}{\mathbb{V}_{\mathbb{Q}}}\left(-\dot{m}_{\mathrm{at}}+\dot{m}_{\mathrm{gp}}+\dot{m}_{\mathrm{EGR}}\right)
$$

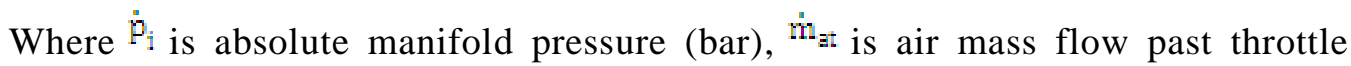
plate $(\mathrm{kg} / \mathrm{sec}), \dot{\mathrm{m}}_{\mathrm{Bp}}$ is air mass flow into intake port $(\mathrm{kg} / \mathrm{sec}), \dot{\mathrm{m}}_{\mathrm{EGR}}$ is EGR mass flow $(\mathrm{kg} / \mathrm{sec}), \mathrm{Ti}$ is intake manifold temperature in Kelvin, $\mathrm{Vi}$ is (manifold \& port passage) volume (m3) and $\mathrm{R}$ is gas constant $(287 \times 10-5)$. The manifold temperature dynamics are described by the differential equation (Hendricks, et al, 2000)

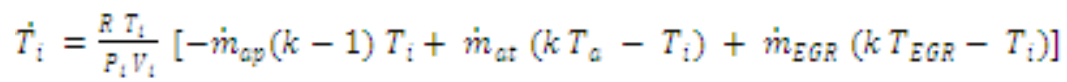

B. Crankshaft Speed Dynamic

The crankshaft dynamics is derived using conservation of rotational energy on the crankshaft (Hendricks, et al, 2000).

$$
\hat{n}=-\frac{1}{d n}\left(P_{f}\left(p_{i}, n\right)+P_{p}\left(p_{i}, n\right)+P_{i}(n)\right)+\frac{1}{d i n} H_{\mathbb{W}} \eta_{i}\left(p_{i}, n_{i} \lambda\right) n_{f}\left(t-\Delta \tau_{d i}\right)
$$

Both the friction power Pf and the pumping power Pp are related with the manifold pressure pi and the crankshaft speed $\mathrm{n}$. The load power $\mathrm{Pb}$ is a function of the crankshaft speed $n$ only. The volumetric efficiency $\eta \mathrm{i}$ is a function of the manifold pressure pi, the crankshaft speed $n$ and the air/fuel ratio AFR. Where I is the scaled moment of inertia of the engine and its load and where the mean injection/torque time delay has been taken into account with the variable $\Delta \tau \mathrm{d}$. 
C. Fuel injection dynamics

According to the identification experiments with an SI engine carried out by Hendricks et al. (Hendricks, et al, 2000), the fuel flow dynamics could be described as

$$
\begin{gathered}
\dot{m}_{f f}=\frac{1}{v_{f}}\left(-\dot{m}_{f f}+X_{f} \dot{m}_{f i}\right. \\
\dot{m}_{f w}=\left(1-X_{f}\right) \dot{m}_{f I} \\
\dot{m}_{f}=\dot{m}_{f w}+\dot{m}_{f f}
\end{gathered}
$$

Where ${ }^{\bar{m}_{f f}}$ is fuel film mass flow, ${ }^{\dot{m}_{f Z}}$ is injected fuel mass flow, ${ }^{\dot{m}_{f v}}$ is fuel vapor mass flow. The model is based on keeping track of the fuel mass flow. The parameters in the model are the time constant $\tau \mathrm{f}$ for fuel evaporation, and the proportion Xf of the fuel which is deposited on the intake manifold or close to the intake valves.

\section{Closed-Loop Control Design}

The Figure 2 shows the block diagram for the automatic control loop for the MVEM including feed-forward and feed-back controllers. Where the MVEM control input $\mathrm{u}$ is the injected fuel mass mfi and the disturbance input $\varnothing$ is the throttle angle position. The feed-forward controller that correlates the steady state value between the MVEM control input mfi and the disturbance $\emptyset$ will be used in the feed-forward path. In order to achieve better transient response, feed-forward and feed-back controller will be designed as following.

\section{A. FF+FB control design}

The feed-forward controller will be implemented by look-up table configuration. The data of this table were determined from the MVEM by using simulation test. The working range for the throttle angle has been given to the MVEM starting from 20 to 60 degree by step 5 degree in order to cover 9 cases. Secondly, the gain $\mathrm{k}$ has been changed for each case to adjust the air fuel ratio equal to 14.7. Finally, the suitable corresponding injected fuel mass can be determined for each throttle angle value by using equation 7 (The data in the look-up-table is omitted here for simplicity).

$$
m f i=k \times \varnothing
$$

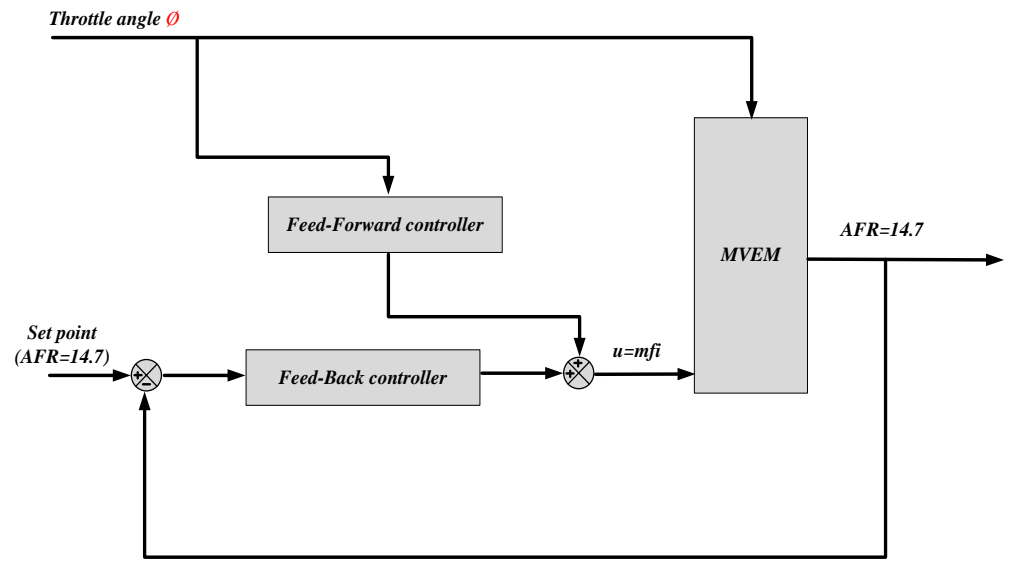

Figure 2. Feed-Forward plus Feedback Control of the MVEM 
The feedback control used a PI controller that is designed using the trial and error method. The design procedure is not presented here due to the limited space.

\section{B. Evaluation}

A new set of square signal as shown in Figure 3 was used as the throttle angle. The range of this excitation signals was bounded between 20 and 60 degrees. This almost covers the whole throttle angle position in normal operation condition. The AFR response of the engine under the developed $\mathrm{FF}+\mathrm{FB}$ control is displayed in Figure $4[10]$. From the Figure 12 it can be seen that the obtained results are very accurate and the AFR equal to 14.7 at the steady state and is acceptable at the transient state. The other outputs of MVEM are crankshaft speed, manifold pressure and temperature. All these outputs will be used for the RBF model training.

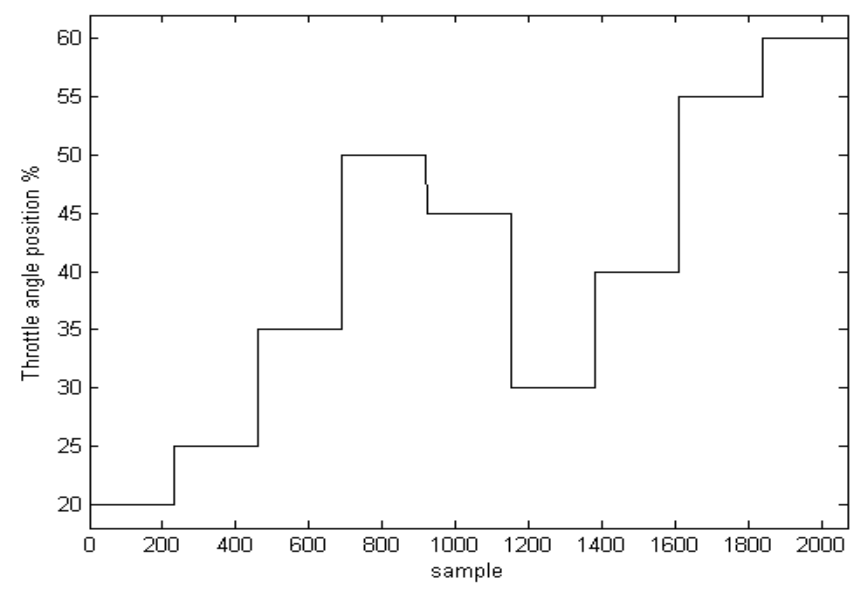

Figure 3. Change of Throttle Angle

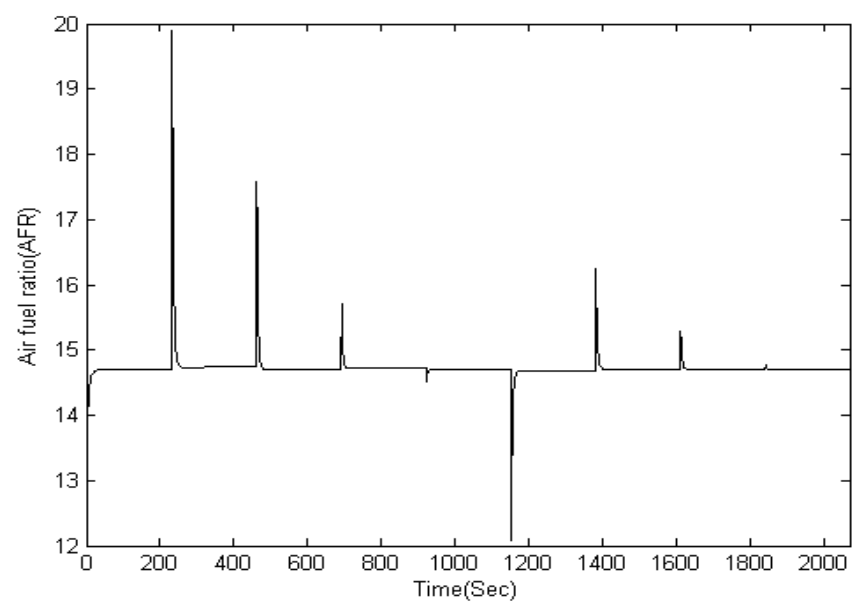

Figure 4. AFR Response under FF+FB Control

\section{Residual Generation}

A. Engine modelling using RBF

A radial basis function (RBF) neural network is used to model the SI engines, because the weights of RBF are linearly related to the objective function, so that any linear 
optimisation algorithm can be used to train the network weights and the training is very fast.

When the Gaussian function is chosen, the output of the network can be calculated according to (8)-(9).

$$
y=W * \phi
$$

where $\mathrm{W}$ is the weight matrix, $\phi \in \mathfrak{R}^{n_{h}}$ is the hidden layer output vector with its $i^{\text {th }}$ entry given as

$$
\varphi_{i}=e^{-\frac{\left\|x-c_{i}\right\|^{2}}{\sigma^{2}}}, \quad i=1, \cdots, n_{h}
$$

where $x$ is the input vector, $c$ is the centre matrix, and $\sigma$ is the width of the Gaussian function. Here centre and width are chosen using the K-means clustering method, while the weights are trained using the Recursive Least Squares algorithm.

There are two different modes of modelling a dynamic system using neural networks, one is the dependent mode and the other is the independent mode. The structure of the independent mode is displayed in Figure 5.

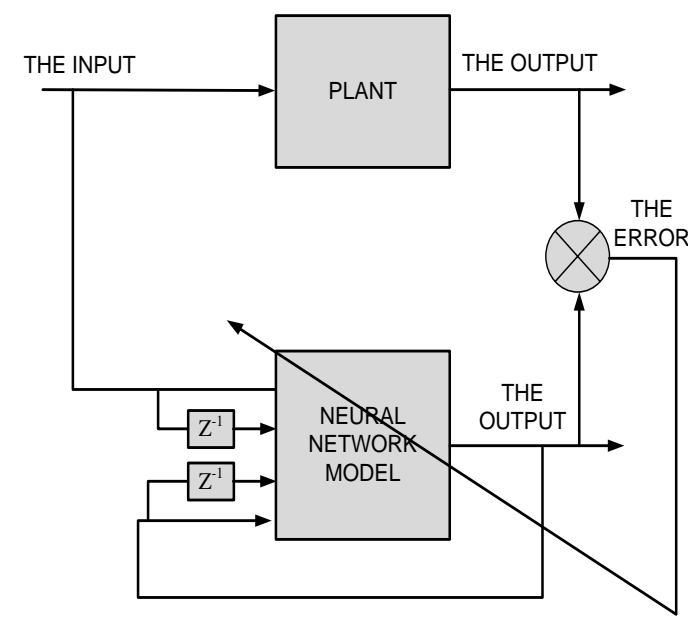

Figure 5. The Structure of Independent Model

In this mode the model output, instead of process output, is fed-back as part of model input in the independent mode. Then, such a model can run independently of the process.

The first step in the engine modelling by using RBFNN is the generation of a suitable training data set. As the training data will influence the accuracy of the neural network modelling performance, the objective of experiment design on training data is to make the measured data become maximally informative, subject to constraints that may be at hand. As mentioned above, a set of random amplitude signals (RAS) were designed for the throttle angle position to obtain a representative set of input data. The sample time of 0.1 sec was used. 


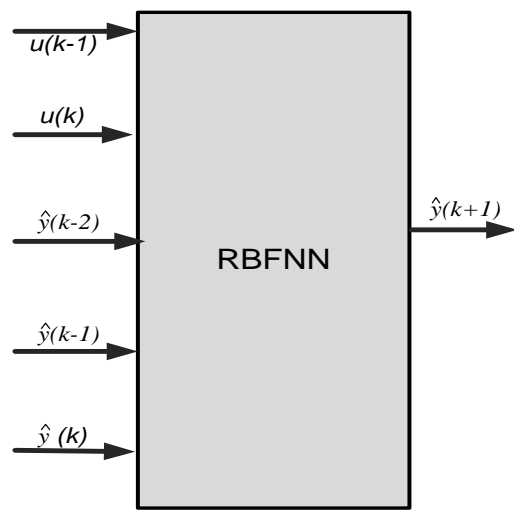

Figure 6. RBFNN Model Structure

The second step is to determine the input variables of the RBF model. The SI engine to be modeled has two input variables: throttle angle and the output of the PID controller which is fuel flow rate, and four outputs: air manifold temperature, air manifold pressure, crank shaft rotary speed and air fuel ratio. According to the dynamics in (1)-(6) and modeling trials, the network input that generated the smallest modeling errors was selected as first order for process input and second order for process output as shown in Figure 6.

As selected above, the RBF model has 16 inputs and 4 outputs. The hidden layer nodes have been selected as 15 . Before the training, 15 centres were chosen using the K-means clustering algorithm, and the width $\sigma$ was chosen using the $p$-nearest-neighbours algorithm. All Gaussian functions in the 15 hidden layer nodes used the same width. For training the weights $W$ the recursive least squares algorithm (Zhai, et al., 2007) was applied and the following initial values were used: $\mu=0.98, w(0)=1.0 \times 10^{-6} \times U_{(n h \times 4) \text {, }}$ $P(0)=1.0 \times 10^{8} \times I_{(\mathrm{nh})}$, where $\mu$ is the forgetting factor, $I$ is an identity matrix and $U$ is the matrix with all element unity, $n_{\mathrm{h}}$ is the number of hidden layer nodes.

Totally a data set with 2070 samples was collected from the MVEM. Before training and testing, the raw data is scaled linearly into the range of [0 1]. Figures 7 10 show the model training and validation results of the 500 samples in the training data set. It can be seen that there is a good match between the two outputs with a very small error, in general. The modelling error of the training data set is smaller than the test data set. The mean absolute error (MAE) index is used to evaluate the modelling effects. For this model the MAE values of crankshaft speed, manifold pressure, manifold temperature and air fuel ratio are $0.0038,0.0942,0.0027$ and 0.0029 respectively.

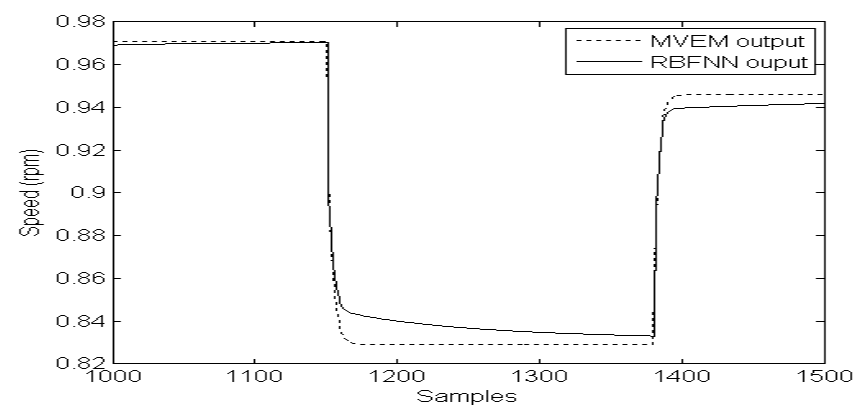

Figure 7. Model and Engine Outputs for Crankshaft Speed 


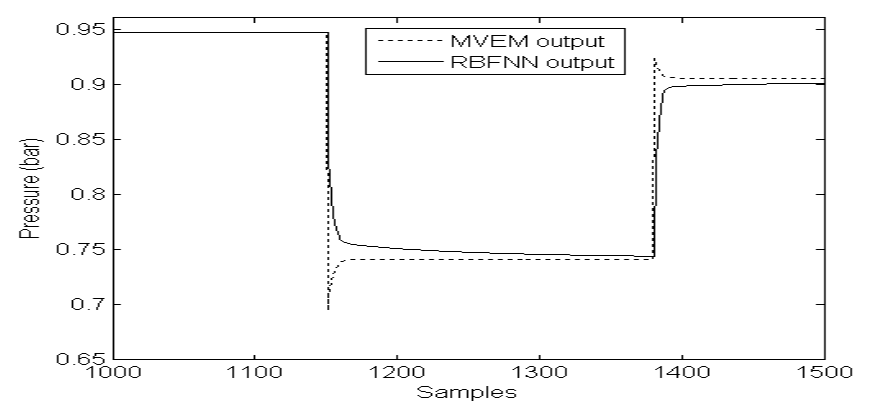

Figure 8. Model and Engine Outputs for Air Manifold Pressure

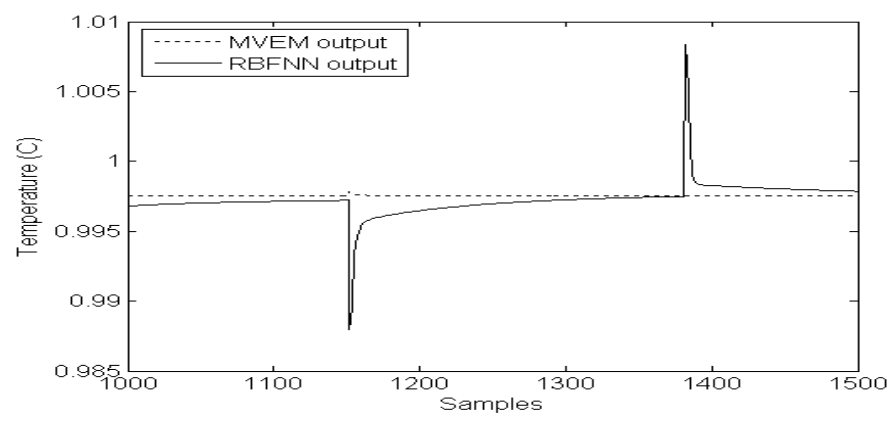

Figure 9. Model and Engine Outputs for Air Manifold Temperature

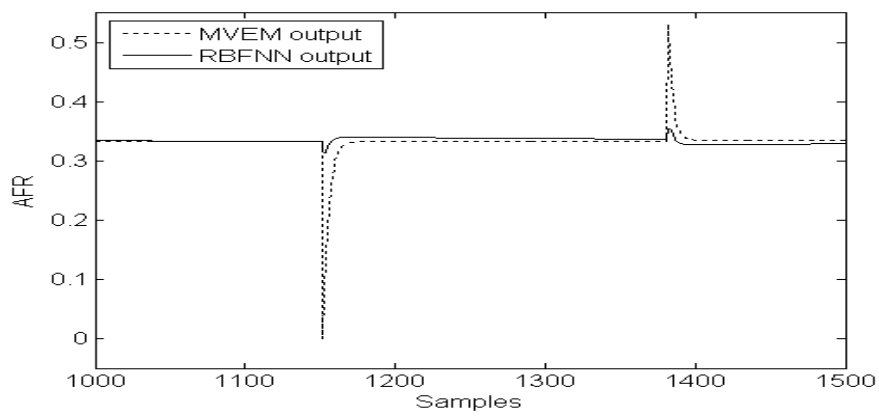

Figure 10. Model and Engine Outputs for AFR

\section{B. Residual Generation}

The whole fault detection and isolation strategy is presented in Figure 18. Firstly, an independent neural network model is trained with engine data collected from the engine without any fault, which is called healthy condition. Then, the model is used parallel to the engine in on-line mode to predict engine output. The modelling error between the engine output and model prediction will be used as residual signal. Thus, if no fault occurs in the engine system, the residual is just modelling error caused by noise and model-plant mismatch. When any fault occurs, the engine output will be affected and will deviate from the nominal values, while the model prediction does not affected by the fault. So, the residual will have a significant deviation from zero caused by faults. The proposed strategy can be applied to different systems to detect dynamic faults. In this study, the residual $\varepsilon$ is generated as the sum-squared filtered modelling error as follows

$$
\begin{gathered}
e(k)=[y(k)-\hat{y}(k)] \\
e_{\text {filtered }}(k)=\mu e_{\text {filtered }}(k-1)+(1-\mu) e(k)
\end{gathered}
$$




$$
\varepsilon(k)=\sqrt{e_{\text {filtered }}^{T} e_{\text {filtered }}}
$$

\section{Application to Engines}

\section{A. Simulating Faults}

Before the developed method is tested on a real engine with real faults, it was tested in this research on the nonlinear simulation of SI engines, the MVEM with different faults simulated on it. One component fault, one actuator fault and four sensor faults with different levels of intensity have been investigated as practical examples of spark ignition (SI) engine faults. The component fault is air leakage in the intake manifold. The actuator fault is a malfunction of the fuel injector. The four sensor faults are malfunction of the intake manifold pressure sensor, manifold temperature sensor, crank shaft speed sensor and air fuel ratio sensor. Details of the simulation of these faults are described as follows.

To collect the engine data subjected to the air leakage fault, equation (1) of the manifold pressure is modified to equation (13):

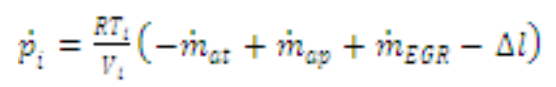

where $\dot{p}_{\mathbb{1}}$ is the absolute manifold pressure (bar), $\dot{m}_{a t}$ is the air mass flow rate past throttle plate $(\mathrm{kg} / \mathrm{sec}), \dot{m}_{\mathrm{gpp}}$ is the air mass flow rate into the intake port $(\mathrm{kg} / \mathrm{sec}), \dot{m}_{\mathbb{E G R}}$ is the EGR mass flow rate $(\mathrm{kg} / \mathrm{sec})$. The added term $\Delta l$ is used to simulate the leakage from the air manifold, which is subtracted to increase the air outflow from the intake manifold. $\Delta l=0$ represents no air leak in the intake manifold. The air leakage level is simulated as $10 \%$ of total air intake in the intake manifold. This fault occurs from the sample number 1450 1500 in the faulty data as shown in Figure 11, and was simulated by changing the Simulink model of the MVEM.

For SI engines, the target is to achieve an air-fuel mixture with a ratio of $14.7 \mathrm{~kg}$ air to $1 \mathrm{~kg}$ fuel. This means the normal value of air fuel ratio is 14.7 . Because any mixture less than 14.7 to 1 is considered to be a rich mixture, any more than 14.7 to 1 is a lean mixture. Lean mixture causes the efficiency of the engine reduced, while rich mixture will cause emission increased. The fuel injector is controlled by the controller with correct amount of fuel. If the fuel injector has any fault the injected fuel amount will not be correct and affect the air/fuel ratio. Here, the malfunction of the fuel inject is simulated by reducing the injected fuel amount of $15 \%$ of the total fuel mass flow rate between the sample number 1750 and 1800 as shown in Figure 11. This fault is also simulated by changing the Simulink model of the MVEM.

The four sensor faults considered are for the crankshaft speed, manifold pressure, manifold temperature and air fuel ratio sensors respectively. 10\%, 15\%, 10\% \& $20 \%$ changes to these sensors are simulated in the MVEM Simulink model with a switch to control its on/off for each fault. These faults are simulated from sample numbers 250 to 300, 550 to 600, 850 to 900 and 1150 to 1200 respectively, as displayed in Figure 11.

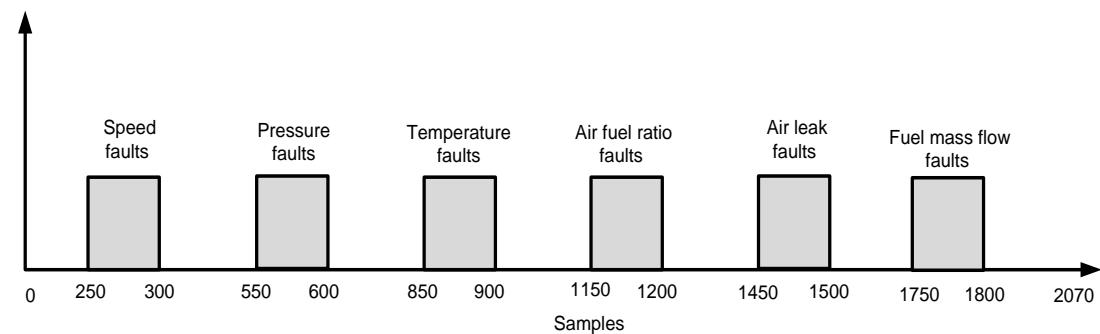

Figure 11. Time Distribution of the Simulated Faults 


\section{B. Fault detection}

The MVEM Simulink model with all 6 faults simulated was run to test the faults, while the throttle angle at different values between $20^{\circ}$ and $60^{\circ}$ for all the fault conditions. The sample time is chosen as $0.02 \mathrm{sec}$ which is the same as that used for engine control.

Figure 12 to Figure 15 show the simulation results for the fault detection of the engine under the closed-loop control. Each figure shows model prediction error of one or two variables. The residuals are calculated from the filtered model prediction errors and are displayed in Figure 16. It is evident that all the 6 simulated faults have been clearly detected, provided not more than one fault will occur simultaneously. Also, there is not a false alarm though the model prediction error is not negligible during the transient states.

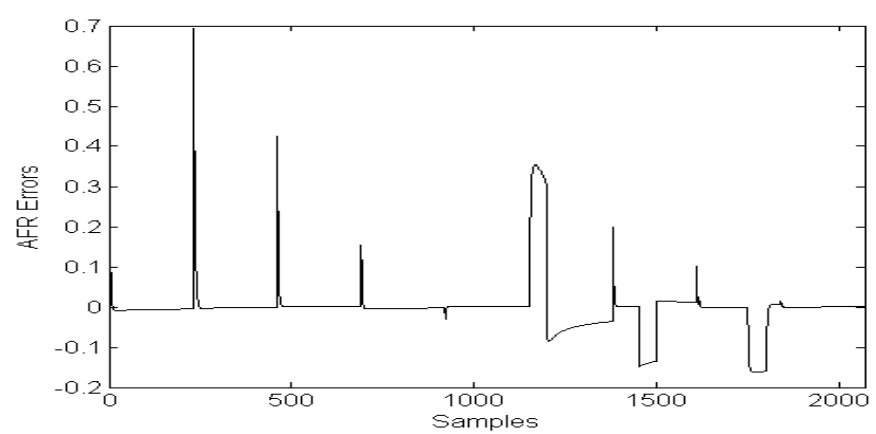

Figure 12. Error of Air Fuel Ratio

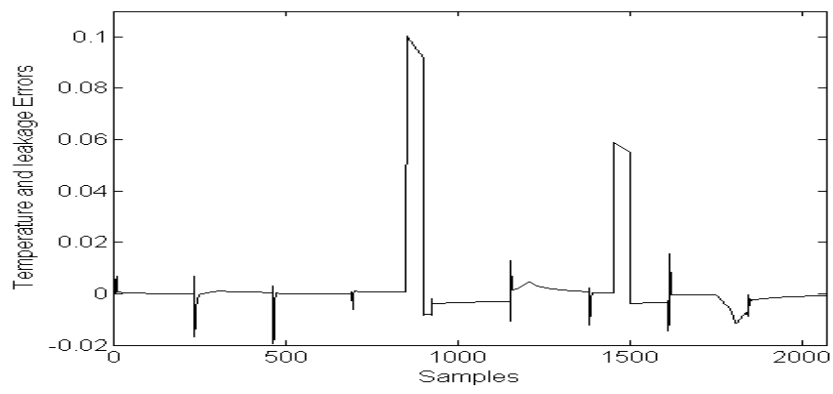

Figure 13. Air Manifold Temperature and Leakage

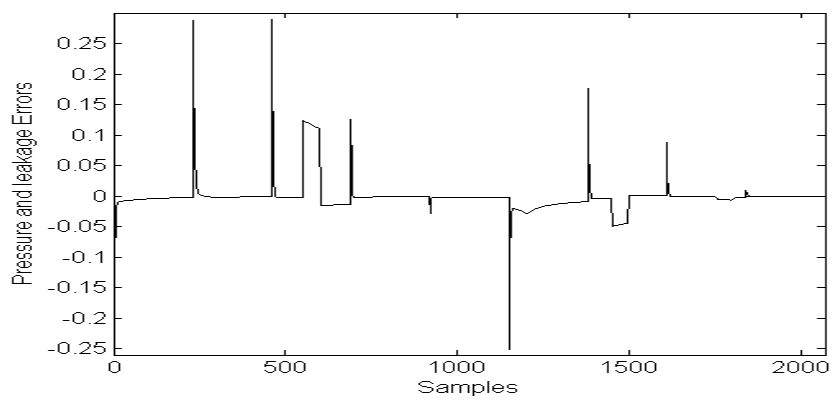

Figure 14. Error of Air Manifold Pressure and Leakage 


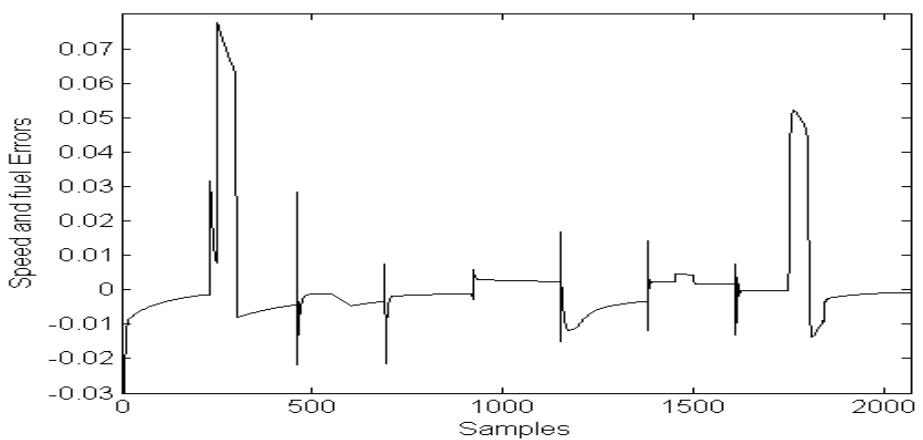

Figure 15. Error of Engine Speed and Fuel

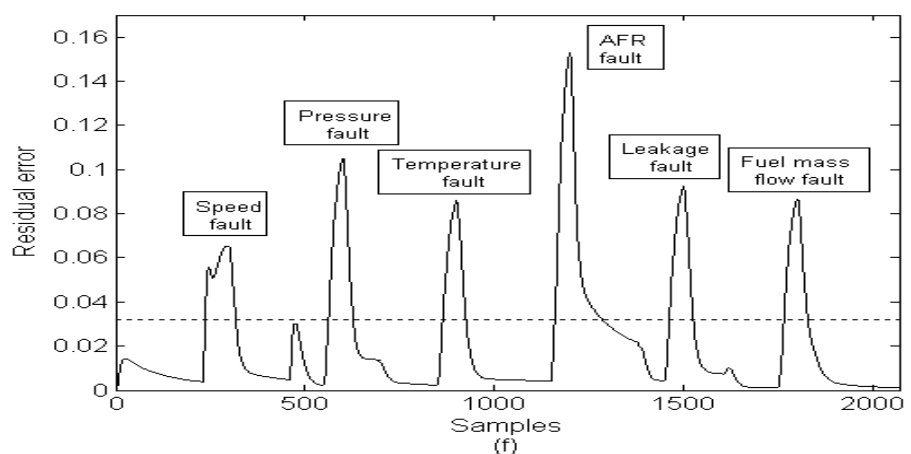

Figure 16. Residuals for All Faults

\section{Conclusions}

Fault detection for closed-loop control system is investigated using neural network model. The training data is acquired when the system is excited by a control variable superimposed with an RAS sequence. In this way the model is more accurate than using the data collected with normal methods. The developed method is evaluated with application to an SI engine nonlinear model. When 6 faults are simulated on the MVEM Simulink model, all the faults are detected with zero false alarm rate and zero fault miss rate. The developed method is possible to be further developed and applied to real engines.

\section{References}

[1] J. Gertler, M. Costin, X. Fang, R. Hira, Z. Kowalczuk and Q. Luo, "Model based on board fault detection and diagnosis for automotive engines", Control Engineering Practice, vol. 1, (1993), pp. 3-17.

[2] J.Gertler, M. Costin, X. Fang, Z. Kowalczuk, M. Kunwer and R. Monajemy, "Model based diagnosis for automotive engines - algorithm development and testing on a production vehicle", IEEE Trans. on Control Systems Technology, vol. 3, (1995), pp. 61-69.

[3] M. Blanke and R.J. Patton, "Industrial actuator benchmark for fault detection and isolation", Control Engineering Practice, vol.3, (1995), pp.1727-17307.

[4] R. Isermann, "Model-based fault-detection and diagnosis status and applications", Annual Reviews in Control, vol.29, (2005), pp.71-85.

[5] D. Capriglione, C. Liguori, C. Pianese and A. Pietrosanto, "On-line sensor fault detection, isolation, and accommodation in automotive engines", IEEE Trans. on instrumentation and measurement, vol. 52, (2003), pp.1182-1189.

[6] D. Capriglione, C. Liguori, C. Pianese and A. Pietrosanto, "Analytical redundancy for sensor fault isolation and accommodation in public transportation vehicles", IEEE Trans. on Instrumentation and Measurement, vol. 53, (2004), pp. 993-999.

[7] D. Capriglione, C. Liguori and A. Pietrosanto, "Real - Time Implementation of IFDIA Scheme in Automotive Systems”, IEEE Trans. on Instrumentation and Measurement, vol. 56, (2007), pp. 824-830. 
[8] E. Hendricks, D. Engler and M. Fam, "A Generic Mean Value Engine Model for Spark Ignition Engines", Proceedings of 41st Simulation Conference, Denmark, DTU Lyngby, (2000).

[9] P. Wang and D.P. Kwok, "Auto-Tuning of Classical PID Controllers Using an Advanced Genetic Algorithm”, IEEE International Conference on Neural Network, (1992).

[10] Y. Feng and X. Jiao, "Transient cylinder air charge estimation with nonlinear Kalman filter for air-fuel ratio control of gasoline engines", Control theory and Application, vol.4, (2015).
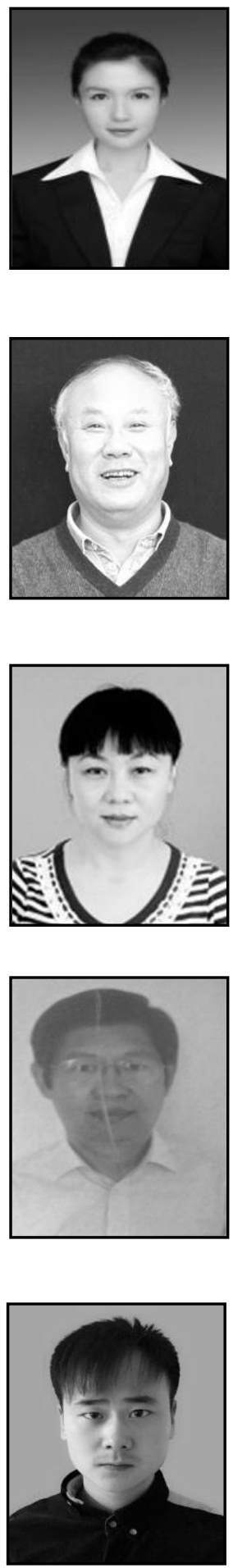

\section{Authors}

Lifei Deng, she is currently a Ph.D student at Jilin University, China. She received her master's degree at Jilin University in 2014.Her research interests are in the fields of signal processing, and engine control theory, system modeling.

Yaowu Shi, he is currently a professor at Jilin University, he is director of the Jilin Institute of automation, senior member of the China Electronics Society, director of the China weak signal detection institute. His research interests are in the fields of signal processing, and engine control theory, system modeling and optimal control theory.

Lanxiang Zhu, she is currently a lecture at Changchun Architecture and Civil engineering College, China. She received her Bachelor (1st class) degree from Jilin Jianzhu University, Master of Science degree from Harbin Institute of Technology, and $\mathrm{PhD}$ degree from Jilin University in 1988, 1998, and 2013 respectively. Her research interests are in the fields of signal processing, and engine control theory, system modeling and optimal control theory.

Dingli Yu, he is currently a professor at Liverpool John Moore University,He received his Bachelor (1st class) degree from Haer Bin Jianzhu University ,Master of Science degree from Jilin University, and PhD degree in 1982, 1986, and 1995 respectively. Dingli $\mathrm{Yu}$ is the member of the international automatic control (IFAC) fault detection and Technical Security Council,the assistant editor for International Journal pattern recognition and control.

Rui Zhu, he is currently an employee at Changchun zonbo new energy transmission system of Limited Liability Company. He received his Bachelor degree from Changchun University of Science and Technology, China. His research interests are in the fields of the mechanical design and its self - moving. 